\title{
Barreras percibidas para la práctica de actividad física en estudiantes universitarios. Diferencias por género y niveles de actividad física*
}

\section{Perceived barriers to Physical Activity in University Students. Differences in Gender and Physical Activity Levels}

\author{
JaVIER SeVIl SERrano \\ Universidad de Zaragoza, España \\ ORCID: http://orcid.org/0000-0001-6972-3088 \\ Alba Práxedes Pizarro \\ Universidad de Extremadura, España \\ Javier Zaragoza CASTERAD \\ Universidad de Zaragoza, España \\ Fernando del Villar Álvarez \\ Universidad Rey Juan Carlos de Madrid, España \\ Luis García-GonzÁlez \\ Universidad de Zaragoza, España
}

\footnotetext{
a Autor de correspondencia. Correo electrónico: jsevils@unizar.es
}

Para citar este artículo: Sevil, J., Práxedes, A., Zaragoza, J., del Villar, F., \& García-González, L. (2017). Barreras percibidas para la práctica de actividad física en estudiantes universitarios. Diferencias por género y niveles de actividad física. Universitas Psychologica, 16(4), 1-15. https://doi.org/10.11144/Javeriana.upsy1 6-4.bppa

\section{RESUMEN}

La etapa universitaria supone un momento sensible respecto a la realización de práctica de actividad física (AF), especialmente en las mujeres. El objetivo principal de este estudio fue identificar la prevalencia de barreras de práctica de AF, y analizar las posibles diferencias en función del género y el cumplimiento de las recomendaciones de práctica de AF para adultos, establecidas por la Organización Mundial de la Salud (2010). Participaron 901 estudiantes universitarios $(M=22.59$; DT $=$ 3.59) que cumplimentaron el IPAQ-SF y la Escala de Percepción de Barreras. Entre los resultados destaca que las barreras relativas a la falta de tiempo obtuvieron los valores más altos entre la población universitaria. El MANOVA realizado mostró que los estudiantes que no cumplían las recomendaciones de práctica de AF percibían valores superiores en la mayoría de barreras de práctica de AF que aquellos estudiantes que sí cumplían las recomendaciones. De igual modo, las mujeres que cumplían las recomendaciones de $\mathrm{AF}$ presentaron valores superiores en todas las barreras analizadas, que los hombres que cumplían dichas recomendaciones. En esta misma línea, las mujeres que no cumplían las recomendaciones de AF presentaron valores superiores en las barreras referentes al tiempo y a la capacidad de disfrute que los hombres que no cumplían dichas recomendaciones. A raíz de la alta prevalencia de percepción de barreras, especialmente en las mujeres, se destaca la importancia de desarrollar programas de intervención y políticas que puedan suponer una mayor adherencia a la práctica de AF, asentando unos hábitos más activos en la población universitaria.

Palabras clave

salud; estilo de vida; barreras; universitarios; actividad física; recomendaciones. 


\begin{abstract}
University stage is a sensitive moment about doing physical activity (PA), especially in women. The main objective of this study was to identify the barriers to PA in university students and analyze possible differences in gender and compliance with PA guidelines established in adults by World Health Organization (2010). 901 students ( $M=$ 22.59; SD = 3.59) completed the IPAQ-SF and the Scale of Perceived Barriers. Among the results, the barriers related to lack of time obtained the highest values among the university population. The MANOVA showed that students who failing to meet PA guidelines perceived higher values in most analyzed barriers than the students who meet PA guidelines. Likewise, women who met the PA guidelines presented higher values in all the barriers analyzed than the men who met the PA guidelines. In addition, women who failing to meet PA guidelines presented higher values in the barriers related to time and disliking of PA than the men who failing to meet PA guidelines. In summary, this study showed a high prevalencia of barriers in university students, especially in women. The results highlight the importance of developing health policies and PA interventions targeting university students.

Keywords

health; lifestyle; barriers; college; physical activity; recommendations.
\end{abstract}

La transición entre la etapa de Educación Secundaria y la Universidad, es un periodo crítico en relación a los niveles de práctica de actividad física (AF) (Cocca, Liukkonen, Mayorga-Vega, \& Viciana-Ramírez, 2014). Atendiendo a la etapa universitaria, el estudio de Haase, Steptoe, Sallis y Wardle (2004), realizado en una muestra de 23 países, señaló que el 39\% de los estudiantes procedentes de países mediterráneos, entre los que se encontraba España, eran inactivos. Concretamente en España, un estudio reciente en universitarios, realizado con acelerometría, mostró que solo el $5.4 \%$ de los estudiantes cumplía las recomendaciones de práctica de AF (Arias-Palencia et al., 2015), establecidas por la Organización Mundial de la Salud (2010), no existiendo diferencias en función del género. Estas recomendaciones señalan que los adultos entre 18 y 64 años, entre los que se encuentran los estudiantes universitarios, deben acumular un mínimo de 150 minutos semanales de $\mathrm{AF}$ aeróbica moderada, o bien un mínimo de 75 minutos semanales de AF aeróbica vigorosa, o una combinación equivalente de AF moderada y/ o vigorosa (AFMV). Sin embargo, otros estudios realizados en estudiantes universitarios españoles (Varela-Mato, Cancela, Ayan, Martín, \& Molina, 2012) y en otros países (Clemente, Nikolaidis, Martins, \& Mendes, 2016), señalaron que las mujeres obtenían unos menores niveles de AFMV y eran más sedentarias. Por tanto, dadas las discrepancias existentes en la literatura en los niveles de AF que realizan hombres y mujeres universitarios, parecen necesarios más estudios que permitan indagar en las variables que dificultan u obstaculizan la práctica habitual de AF en ambos sexos.

En este ámbito de actuación, el Modelo Social-Ecológico (Sallis, Owen \& Fisher, 2008; Spence \& Lee, 2003) intenta explicar cuáles son los factores que influencian la práctica de AF (Yan, Voorhees, Beck, \& Wang, 2014), conjugando así los factores individuales del sujeto (e.g. psico-sociales, personales, barreras para la realización de $\mathrm{AF}$ ) y los factores referidos al contexto (e.g. políticas saludables; Keating, Guan, Piñero, \& Bridges, 2005). De los factores individuales, la literatura científica muestra que la identificación de las barreras para la realización de la $\mathrm{AF}$, debe ser uno de los objetivos prioritarios para facilitar una conducta más activa entre los universitarios (Serrano-Sánchez, LeraNavarro, Dorado-García, González-Henriquez, \& Sanchis-Moysi, 2012). En este sentido, algunos estudios en población universitaria encontraron que las barreras para la realización de práctica de $\mathrm{AF}$ se relacionan negativamente con los niveles de AFMV (e.g. Ferreira, Aparecido, \& Rodrigues, 2012). Por otro lado, Gyurcsik, Spink, Bray, Chad y Kwan (2006) señalaron que las barreras para la práctica de AF aumentaban su frecuencia y variaban su tipología desde el instituto hasta la etapa universitaria. Este hecho podría deberse al cambio en el estilo de vida que puede producirse en el tránsito de la educación secundaria a la etapa universitaria (e.g. abandono de las clases de Educación Física, cambio de residencia, estrés, falta de apoyo social, aumento en las alternativas de ocio) (Deliens, Deforche, De Bourdeaudhuij, \& Clarys, 2015). Todo ello, podría provocar un descenso de los 
niveles de AFMV y un aumento en la percepción de las barreras de práctica de AF (Bray, 2007; Gyurcsik et al., 2006), especialmente entre las mujeres universitarias (Han et al., 2008).

En estos últimos años, varios estudios han utilizado diversas clasificaciones para examinar las barreras específicas para la realización de $\mathrm{AF}$ en los diferentes grupos de población (i.e. niños, adolescentes, jóvenes-adultos, adultos, personas mayores). De este modo, algunos estudios han optado por diferenciar entre barreras internas (i.e. cuestiones personales e individuales) o externas al sujeto (i.e. cuestiones que afectan al contexto de práctica; e.g. Gunnell, Brunet, Wing, \& Bélanger, 2015; Zaragoza, Generelo, Julián, \& Abarca-Sos, 2011).

Concretamente, existen estudios en el ámbito universitario, que han centrado su objetivo en esclarecer las barreras que inhiben la práctica de AF (e.g. Ramírez-Vélez et al., 2015) especialmente en las mujeres (Lovell, El Ansari \& Parker, 2010). Un estudio reciente en España, revela que la principal barrera para adoptar un estilo de vida más activo en los estudiantes universitarios es la falta de tiempo (Martínez-Lemos, Puig-Ribera, \& García-García, 2014) lo que puede llevar en muchos casos al abandono de la práctica de AF (GómezLópez, Granero-Gallegos, Baena-Extremera, \& Ruiz-Juan, 2011). Sin embargo, en otros estudios las barreras internas como la falta de interés, la poca utilidad o motivación, o los sentimientos de pereza, apatía o incompetencia percibida emergen como posibles barreras para la práctica de AF en esta etapa (Ashton, Hutchesson, Rollo, Morgan, \& Collins, 2016; Daskapan, Tuzun, \& Eker, 2006; Gómez-López, Granero-Gallegos, \& Baena-Extremera, 2010). En otros países los estudiantes universitarios también señalan como principales barreras para la práctica de AF el mal tiempo, la falta de dinero o de instalaciones para practicar AF (El-Gilany, Badawi, El-Khawaga, \& Awadalla, 2011; Ibrahim, Karim, Oon, \& Ngah, 2013; Reichert, Barros, Domingues, \& Hallal, 2007).

Sin embargo, existe un número limitado de estudios que hayan analizado las barreras para la práctica de AF en estudiantes universitarios, estableciendo diferencias en función del género (e.g. Ramírez-Vélez et al., 2015) y los niveles de AF (e.g. Awadalla et al., 2014). En este sentido, un estudio de Hazam (2013) señaló que las mujeres universitarias perciben generalmente más barreras externas (e.g. falta de tiempo), mientras los hombres reportan más barreras internas (e.g. falta de motivación). Sin embargo, un estudio reciente de Ramírez-Vélez et al. (2015) mostró que las mujeres presentaban una mayor prevalencia de barreras que los hombres, en todos los factores analizados (i.e. falta de tiempo, influencia social, falta de energía, falta de voluntad, falta de habilidades y falta de recursos), salvo en el miedo a lesionarse, en el que los hombres presentaban valores ligeramente superiores que las mujeres. Por otro lado, Awadalla et al. (2014) analizaron las barreras de prácticas de AF, estableciendo diferencias en función del cumplimiento de las recomendaciones de la OMS (2010) sobre los estudiantes universitarios. En este último estudio, los participantes que no cumplían las recomendaciones de $\mathrm{AF}$, reportaron valores significativamente superiores en la mayoría de barreras analizadas. No obstante, en algunas barreras como la falta de condición física, el clima atmosférico o las malas experiencias deportivas, no se obtuvieron diferencias entre los dos grupos.

Por tanto, dada la variedad de resultados encontrados en la literatura, el objetivo principal de este estudio es identificar la prevalencia de barreras de práctica de AF, y analizar las posibles diferencias en función del género y el grado del cumplimiento de las recomendaciones internacionales de AF establecidas por la OMS (2010). Se plantea como hipótesis inicial que las principales barreras para la realización de AF entre los estudiantes universitarios serán aquellas relativas al tiempo. La segunda hipótesis sostiene que los estudiantes universitarios que no cumplen las recomendaciones de práctica de AF establecidas por la OMS (2010) percibirán más barreras para realizar $\mathrm{AF}$ que aquellos que sí que cumplen las recomendaciones establecidas. Finalmente, la tercera hipótesis señala que las mujeres universitarias percibirán más barreras de práctica de AF que los hombres universitarios. 
Javier Sevil Serrano, Alba Práxedes Pizarro, Javier Zaragoza Casterad, Et al.

\section{Método}

\section{Participantes}

El estudio se realizó en Cáceres, una provincia situada al oeste de España, al norte de la Comunidad Autónoma de Extremadura, que cuenta con 95855 habitantes y una extensión de $1750.33 \mathrm{~km}^{2}$. De una muestra total de 10279 estudiantes universitarios matriculados en el curso 2013/2014 en el Campus de Cáceres, se realizó un muestreo no probabilístico por conveniencia, tomando en consideración el género, el curso y los estudios realizados. Participaron un total de 901 estudiantes universitarios, 408 hombres (M $=22.61 ; D T=3.73)$ y 493 mujeres $(M=$ 22.58; $\mathrm{DT}=3.47$ ), con edades comprendidas entre los 18 y 49 años $(M=22.59$; $D T=$ 3.59). Concretamente, 270 estudiantes tenían entre 18 y 20 años, 355 entre 21 y 23 años y 276 entre 24 años y 49 años. De los 901 estudiantes, 680 eran estudiantes de grado y 221 estudiantes de posgrado. La distribución de los estudiantes por titulación académica fue la siguiente: 188 de Magisterio Infantil, 237 de Magisterio de Primaria, 255 de Ciencias de la Actividad Física y del Deporte, 32 del Posgrado en Educación Física, 31 del Posgrado en Geografía e Historia, 23 del Posgrado de Lengua y Literatura, 24 del Posgrado de Ciencias de la Salud, 24 del Posgrado de Intervención Sociocomunitaria, 30 del Posgrado de Lenguas Extranjeras, 25 del Posgrado de Informática y 33 del Posgrado de Matemáticas. La distribución de los estudiantes por curso académico fue la siguiente: 223 estudiantes de primer curso, 211 estudiantes de segundo curso, 201 estudiantes de tercer curso, 45 estudiantes de cuarto curso y 221 estudiantes de quinto curso (posgrado). Los sujetos fueron informados de los objetivos del estudio, y dieron su consentimiento por escrito de manera anónima y voluntaria. El Comité de Ética de la Universidad de Extremadura aprobó la realización del estudio.
Variables del estudio e instrumento

\section{Barreras para realizar $\mathrm{AF}$}

Para medir la percepción de barreras para la práctica de AF, se utilizó la Escala de Percepción de Barreras (Chinn, White, Harland, Drinkwater, \& Raybould, 1999), utilizada previamente en población adolescente española (Zaragoza et al., 2011). Este instrumento está compuesto por 17 ítems que se estructuran en varios factores: capacidad de disfrute (ocho ítems; e.g. "No soy bueno en las actividades físicas y/o deportivas"), tiempo (cuatro ítems; e.g. "Tengo demasiados trabajos en la universidad"), seguridad (un ítem; e.g. "No es seguro estar haciendo ejercicio físico en la calle, barrio, parque...") y el contexto de práctica (tres ítems; e.g. "No tengo a nadie con quien realizar actividad física"). La Escala está encabezada por el enunciado "Contesta si las siguientes razones son para ti un problema para hacer ejercicio". El formato de respuesta empleado estaba indicado en una escala Likert de cero a seis, donde el cero correspondía a "No es en absoluto un problema" y el seis a "Es una problema grave". Los estudiantes que respondían las barreras con un número igual o superior a 1 , son aquellos que percibían esa barrera para la realización de AF, mientras que los que contestaban 0 , no tenían ese problema (Zaragoza et al., 2011).

Debido a que esta Escala no ha sido validada en población universitaria, se realizó un análisis factorial exploratorio (AFE) para evaluar las características psicométricas del instrumento. De los cuatro factores iniciales, se eliminó el factor "seguridad" porque solo medía un ítem, y el peso factorial se agrupaba, tras el análisis, con el factor relativo al "contexto de práctica". Por tanto, se realizó una nueva agrupación escogiendo los ítems de los factores de "seguridad" y del "contexto de práctica". Así, el nuevo factor resultante "barreras de contexto y seguridad en la práctica", compuesto por cuatro ítems, medía las barreras relativas a estos dos factores iniciales. Los tres factores obtenidos explicaron el 60\% de la varianza total. Las barreras relativas a 
la capacidad de disfrute, con un peso factorial entre 0.42 y 0.865 explicaron el $14 \%$ de la varianza. Por otro lado, las barreras relativas a la falta de tiempo, con un peso factorial entre 0.784 y 0.86 explicaron el $19 \%$ de la varianza. Por último, las barreras relativas al contexto y seguridad en la práctica, con un peso factorial entre 0.65 y 0.754 explicaron el $14 \%$ de la varianza. Se obtuvieron coeficientes alfa de Cronbach de 0.87 para las barreras referentes a la capacidad de disfrute, de 0.87 para las barreras relativas al tiempo, y de 0.81 para las barreras de contexto y seguridad en la práctica de AF. El análisis factorial confirmatorio (AFC) reveló en el presente estudio unos índices de ajuste adecuados para los tres factores propuestos en la escala $\left(\chi^{2}(142)=342.51, \mathrm{p}<0.01 ;\right.$ RMSEA $=$ 0.06; CFI $=0.93$; SRMR $=0.06)$.

\section{Niveles de AF}

Para conocer los niveles de AF en estudiantes universitarios, se utilizó el International Physical Activity Questionnaire-Short Form (IPAQ-SF; Booth, 2000), validado en población española (Román, Ribas, Ngo, \& Serra, 2013), y utilizado ampliamente a nivel internacional. Este cuestionario ha sido validado en 12 países (Craig et al., 2003), lo que demuestra adecuadas propiedades psicométricas para medir los niveles de AF que realizan los estudiantes universitarios (e.g Rodríguez-Muñoz, Corella, Abarca-Sos, \& Zaragoza, 2017). Para ello, se escogieron las cuatro primeras preguntas, de las siete que componen este cuestionario, ya que son las que permiten cuantificar los niveles de AF de intensidad moderada y vigorosa, y calcular el cumplimiento de las recomendaciones de AF. En el cuestionario, los estudiantes registraron el número de minutos y horas diarias de $\mathrm{AF}$ moderada y vigorosa, así como el número de días a la semana de práctica de $\mathrm{AF}$ a estas dos intensidades. Siguiendo las recomendaciones internacionales de la OMS (2010), los adultos entre 18 y 64 años deben acumular un mínimo de 150 minutos semanales de AF aeróbica moderada, o bien un mínimo de 75 minutos semanales de AF aeróbica vigorosa, o bien una combinación equivalente de AFMV. Para ello, se calcularon los niveles de AF moderada y AF vigorosa semanales de los estudiantes, a través de la suma de los minutos diarios a estas intensidades y los días de práctica de AF. Atendiendo a estas recomendaciones de la OMS (2010), en el presente estudio se categorizó el cumplimiento de práctica de AFMV en una escala dicotómica (i.e. cumplen o no cumplen las recomendaciones).

\section{Diseño y procedimiento}

Se realizó un estudio de carácter descriptivo y transversal, mediante una metodología de investigación de carácter cuantitativo. Para ello, en primer lugar, se contactó con los diferentes docentes universitarios, con la finalidad de informar sobre los objetivos del estudio, y se establecieron varias fechas y aulas para la administración de los diferentes cuestionarios. El investigador principal estuvo presente en la toma de datos, lo que garantizó el anonimato de las respuestas. Los estudiantes cumplimentaron los cuestionarios individualmente, en un clima de trabajo adecuado, en el que destinaron un tiempo aproximado de 10 minutos. El estudio tuvo lugar en el periodo de clases durante el mes de marzo de 2014, lejos del periodo de exámenes.

\section{Análisis de datos}

Los distintos análisis se realizaron a través del software estadístico SPSS 21.0. En primer lugar, a través de la prueba de Kolmogorov-Smirnov, se determinó que las variables del estudio seguían una distribución normal. Posteriormente, se llevó a cabo un AFE, a través del método Varimax, de la Escala de Percepción de Barreras con el objeto de conocer la estructura factorial de los ítems, así como un AFC para confirmar la estructura factorial de la escala. De igual modo, se efectuaron cálculos de la consistencia interna, a través del coeficiente de alfa de Cronbach, así como los estadísticos descriptivos (media y desviación típica) de cada factor. Se realizó un análisis de 
regresión de pasos sucesivos, considerando como variable dependiente los niveles de AFMV, y como variables independientes los tres factores del cuestionario de barreras. De igual modo, se efectuó un análisis de regresión logística binomial, tomando como variable dependiente el cumplimiento de las recomendaciones de práctica de AF, y como variables independientes los tres factores de barreras. Finalmente, el análisis de varianza multivariante (MANOVA) con dos factores (género x cumplimiento de las recomendaciones) permitió valorar, a través del test post-hoc de Bonferroni, si existían diferencias significativas en las diferentes barreras para la realización de $\mathrm{AF}$ en función del género y del cumplimiento de las recomendaciones de práctica de AF. Para ello, se calculó también el tamaño del efecto a través del estadístico eta cuadrado parcial $\left(\eta^{2} \mathrm{p}\right)$, el cual permite conocer la magnitud de las diferencias encontradas al eliminar la influencia del tamaño de la muestra, así como el intervalo de confianza al 95\% para las diferencias.

\section{Resultados}

En relación al cumplimiento de las recomendaciones de práctica, se puede observar en la tabla 1, que un $58 \%$ de los estudiantes alcanzaron las recomendaciones establecidas por la OMS (2010). Por otro lado, los niveles de AFMV se sitúan en 279.52 minutos semanales. Atendiendo al género, los hombres obtienen un mayor cumplimiento de práctica de AF (76.7\%) y unos mayores niveles de AFMV semanales (384.93) que las mujeres.

\section{TABLA 1}

Estadísticos descriptivos del cumplimiento de las recomendaciones de práctica de AF y los niveles de $A F$ en hombres y mujeres universitarios

\begin{tabular}{|c|c|c|c|c|c|c|c|c|}
\hline & \multicolumn{2}{|c|}{$\begin{array}{l}\text { Total de } \\
\text { estudiantes }\end{array}$} & \multicolumn{2}{|c|}{$\begin{array}{l}\text { Cumplen las } \\
\text { recomendaciones }\end{array}$} & \multicolumn{2}{|c|}{$\begin{array}{l}\text { No cumplen las } \\
\text { recomendaciones }\end{array}$} & \multicolumn{2}{|c|}{$\begin{array}{l}\text { Niveles de AFMV } \\
\text { semanales }\end{array}$} \\
\hline & $\mathrm{N}$ & $\%$ & $\mathrm{~N}$ & $\%$ & $\mathrm{~N}$ & $\%$ & $\mathrm{M}$ & DT \\
\hline Hombres & 408 & $45.28 \%$ & 313 & $76.7 \%$ & 95 & $23.3 \%$ & 384.93 & 315.21 \\
\hline Mujeres & 493 & $54.71 \%$ & 211 & $42.8 \%$ & 282 & $57.2 \%$ & 192.47 & 340.68 \\
\hline Total & 901 & & 524 & $58.2 \%$ & 377 & $41.8 \%$ & 279.62 & 342.88 \\
\hline
\end{tabular}

En relación a las barreras para la realización de AF, se puede apreciar en la tabla 2 y 5 , que los ítems relativos a la falta de tiempo obtuvieron los valores más altos entre la población universitaria $(\mathrm{M}=3.34)$. En este sentido, los siguientes ítems "Tengo demasiados trabajos en la universidad" (92.34\%) y "Tengo que estudiar mucho" (89.9\%) fueron percibidos como las principales barreras para realizar AF. Seguidamente, se situaron las barreras contextuales y de seguridad en la práctica (M $=1.27$ ), obteniendo en las barreras relativas a "No tengo el equipo necesario para hacer actividad física" (52.83\%) y "No tengo a nadie con quien realizar actividad física" (51.16\%), las puntuaciones más altas. Por último, las barreras relativas al disfrute $(\mathrm{M}=1.07)$ obtuvieron los valores más bajos de los tipos de barreras analizados. Las barreras de "Soy muy perezoso/a para hacer actividad física" (62.81\%), "Por lo general estoy cansado/a para hacer actividad física" (55.49\%) y "No soy bueno en las actividades físicas $y / 0$ deportivas" (53.05\%) fueron identificadas con mayor frecuencia en la población estudiada. 
TABLA 2

Prevalencia de barreras para la realización de AF

\begin{tabular}{|c|c|c|}
\hline \multirow[t]{3}{*}{ Barreras para la realización de $\mathrm{AF}$} & \multirow{2}{*}{\multicolumn{2}{|c|}{$\begin{array}{c}\text { Estudiantes } \\
(\mathbf{n}=901)\end{array}$}} \\
\hline & & \\
\hline & $\mathrm{N}(\%)$ & $\mathrm{M}(\mathrm{DT})$ \\
\hline $\begin{array}{l}\text { 1. No hay un buen sitio para hacer } \\
\text { actividad física }\end{array}$ & $\begin{array}{l}400 \\
(44.39)\end{array}$ & $\begin{array}{l}1.15 \\
(1.6)\end{array}$ \\
\hline $\begin{array}{l}\text { 2. No tengo a nadie con quien realizar } \\
\text { actividad física. }\end{array}$ & $\begin{array}{l}461 \\
(51.16)\end{array}$ & $\begin{array}{l}1.49 \\
(1.86)\end{array}$ \\
\hline 3. No tengo el equipo necesario para & 476 & 1.46 \\
\hline hacer actividad física & $(52.83)$ & $(1.78)$ \\
\hline 4. No es seguro estar haciendo ejercicio & 413 & 0.99 \\
\hline 5. No tengo suficiente tiempo & $\begin{array}{l}735 \\
(81.57)\end{array}$ & $\begin{array}{l}3.19 \\
(2.11)\end{array}$ \\
\hline $\begin{array}{l}\text { 6. Tengo demasiados trabajos en la } \\
\text { universidad }\end{array}$ & $\begin{array}{l}832 \\
(92.34)\end{array}$ & $4(1.93)$ \\
\hline $\begin{array}{l}\text { 7. Tengo muchas tareas que realizar en } \\
\text { casa }\end{array}$ & $\begin{array}{l}781 \\
(86.68)\end{array}$ & $\begin{array}{l}2.89 \\
(1.86)\end{array}$ \\
\hline 8. Tengo que estudiar mucho & $\begin{array}{l}810 \\
(89.9)\end{array}$ & $\begin{array}{l}3.29 \\
(1.81)\end{array}$ \\
\hline 9. No me gusta la actividad física & $\begin{array}{l}379 \\
(42.06)\end{array}$ & $\begin{array}{l}0.89 \\
(1.38)\end{array}$ \\
\hline $\begin{array}{l}\text { 10. Por lo general estoy cansado ja para } \\
\text { hacer actividad física }\end{array}$ & $\begin{array}{l}500 \\
(55.49)\end{array}$ & $\begin{array}{l}1.41 \\
(1.64)\end{array}$ \\
\hline 11. No disfruto de la actividad física & $\begin{array}{l}386 \\
(42.84)\end{array}$ & $\begin{array}{l}0.91 \\
(1.38)\end{array}$ \\
\hline $\begin{array}{l}\text { 12. No soy bueno en las actividades } \\
\text { físicas y jo deportivas }\end{array}$ & $\begin{array}{l}478 \\
(53.05)\end{array}$ & $\begin{array}{l}1.33 \\
(1.69)\end{array}$ \\
\hline 13. Me da miedo lesionarme & $\begin{array}{l}385 \\
(42.73)\end{array}$ & $\begin{array}{l}0.95 \\
(1.44)\end{array}$ \\
\hline $\begin{array}{l}\text { 14. Soy muy perezosola para hacer } \\
\text { actividad física. }\end{array}$ & $\begin{array}{l}566 \\
(62.81)\end{array}$ & $\begin{array}{l}1.81 \\
(1.9)\end{array}$ \\
\hline 15. Hacer actividad física es aburrido & $\begin{array}{l}390 \\
(43.28)\end{array}$ & $\begin{array}{l}0.85 \\
(1.31)\end{array}$ \\
\hline $\begin{array}{l}\text { 16. Me pongo nervioso /a cuando hago } \\
\text { actividad física. }\end{array}$ & $\begin{array}{l}270 \\
(29.96)\end{array}$ & $\begin{array}{l}0.49 \\
(0.93)\end{array}$ \\
\hline
\end{tabular}

Notas: $\mathrm{N}(\%)$ = Número y porcentaje de estudiantes que señalan ese ítem como una barrera para la realización de AF $\mathrm{M}=$ Media DT $=$ Desviación Típica

Fuente: elaboración propia.

Por otro lado, los resultados del análisis de regresión lineal por pasos sucesivos para los niveles de AFMV semanales indicaron que las barreras relativas al desagrado de la AF predecían un $12.1 \%$ de la varianza explicada (unstandardized beta $=-0.100 .37$, standard error $=9.93$, standardized beta $=-0.348, \mathrm{p}<0.001$ ), mientras que las barreras relativas al tiempo predecían un $2.5 \%$ de la varianza explicada (unstandardized beta $=-0.35 .3$, standard error $=6.93$, standardized beta $=-0.16, \mathrm{p}<0.001$ ). Asimismo, los resultados del análisis de regresión logística binaria indicaron que las barreras relativas al desagrado de la $\mathrm{AF}(\mathrm{B}=-0.93, \mathrm{SE}=$ $0.09, \operatorname{Exp}[B]=0.39, p<0.001)$ y las barreras relativas al tiempo $(\mathrm{B}=-0.35, \mathrm{SE}=0.05, \mathrm{Exp}[\mathrm{B}]$ $=0.7, \mathrm{p}<0.001)$ predecían significativamente el cumplimiento de las recomendaciones de AF.
Los resultados del MANOVA (tabla 3) mostraron un efecto significativo del cumplimiento de las recomendaciones de práctica de AF sobre las diferentes barreras de práctica de AF (Lambda de Wilks = 0.827; $F(62.235)=p<0.001 ; \eta 2 p=0.173)$. La prueba del post-hoc de Bonferroni mostró que aquellos estudiantes que no cumplían las recomendaciones de práctica de AF obtuvieron valores significativamente superiores en todas las barreras de práctica, a excepción de las barreras: "No hay un buen sitio para hacer actividad física" y "No es seguro estar haciendo ejercicio físico en la calle, barrio, parque" (ver tabla 3). Por otro lado, en este MANOVA (tabla 4 y 5) se apreció un efecto de interacción significativo del género y del cumplimiento de las recomendaciones de AF sobre las barreras para su realización. En el género masculino, se obtuvieron diferencias significativas en las barreras de práctica de $\mathrm{AF}$ en función del cumplimiento de las recomendaciones de práctica (Lambda de Wilks $\left.=0.918 ; \mathrm{F}(26.697)=\mathrm{p}<0.001 ; \eta^{2} \mathrm{p}=0.082\right)$. En el género femenino igualmente se obtuvieron diferencias significativas en las barreras de práctica de $\mathrm{AF}$, en función del cumplimento de las recomendaciones de práctica (Lambda de Wilks $=0.885 ; \mathrm{F}(38.843)=\mathrm{p}<0.001 ; \eta^{2} \mathrm{p}$ $=0.115)$. La prueba del post-hoc de Bonferroni mostró que tanto los hombres como mujeres que no cumplían las recomendaciones de práctica, percibían más barreras para la práctica de $\mathrm{AF}$ (ver tabla 4).

\section{TABLA 3}

Prevalencia de barreras para la realización de AF y análisis de diferencias en función del cumplimiento de las recomendaciones de práctica de AF y del género

\begin{tabular}{|c|c|c|c|c|c|c|c|}
\hline \multirow[t]{2}{*}{ Barreras para la realización de AF } & \multicolumn{2}{|c|}{$\begin{array}{c}\text { Cumplen las } \\
\text { recomendaciones } \\
(\mathrm{n}=524)\end{array}$} & \multicolumn{2}{|c|}{$\begin{array}{c}\text { No cumplen las } \\
\text { recomentaciones } \\
(n=37)\end{array}$} & \multicolumn{3}{|c|}{ Contraste entre grupos } \\
\hline & & $M(D)$ & & $M(D T)$ & & & \\
\hline No hay un buen stio para hacer actiridad fisica & $\frac{163(41.2)}{216}$ & $\frac{1.071(1.58)}{1.078}$ & & $1.26(1.63)$ & 2.961 & & \\
\hline Vo & $218(41.6)$ & $11(16)$ & & & & 0.063 & $<0.001$ \\
\hline 3. No tengo elequipo necesario para hacer actividad fisia & 239 (45.6) & $1.17(1.61)$ & $235(62.9)$ & $1.87(1.92)$ & 35.334 & 0.038 & $<0.001$ \\
\hline 4. No es seguro estar haciendo cejercicio friso en la calle, bario, parque. & $228(43.5)$ & $0.91(1.35)$ & $185(49.1)$ & $1.09(1.47)$ & 3.25 & 0.004 & 0.072 \\
\hline 5. No tengo sulfiente tiempo & $402(76.7)$ & $258(1.97)$ & $333(88.3)$ & $4.03(2.01)$ & 115.273 & 0.109 & \\
\hline 6. Tengo demasiados trabajios en la universidad & $472(90.1)$ & $3.52(1.22)$ & 359 (95.5) & $4.66(1.72)$ & 82.931 & 0.083 & $<0.001$ \\
\hline 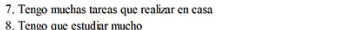 & $434(82.8)$ & $241(1.71)$ & $346(92)$ & $3.55(1.86)$ & 90.424 & 0.089 & $<0.001$ \\
\hline 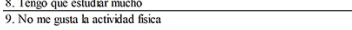 & $\begin{array}{llll}4 & 459(78.6) \\
142(27.1)\end{array}$ & $\begin{array}{l}2.91(1.15) \\
0.44(0.93)\end{array}$ & 2351(193.1) & & $\frac{59.202}{15041}$ & 0.062 & $\frac{<.001}{<<.001}$ \\
\hline 10. Por lo general cstoy canssado/ para hacer acividad fisica & $228(43.5)$ & $0.89(1.28)$ & $272(72.1)$ & $2.14(1.79)$ & 149.298 & 0.14 & $<0.001$ \\
\hline 11. No disfüo de ba actividad fsica & 150 (28.6) & $0.43(0.83)$ & $136(62.6)$ & $1.57(1.69)$ & 178.731 & 0.167 & $<0.001$ \\
\hline 12. No soy bueno en las actividades fisicas y'o deportivas & $199(38)$ & $0.73(1.21)$ & $279(84)$ & $2.16(1.89)$ & 189.242 & 0.175 & $<0.001$ \\
\hline 13. Me da miedo ksionarame & $200(38.2)$ & $0.84(1.37)$ & $185(49.1)$ & $1.09(1.52)$ & 201.245 & 0.007 & $<0.001$ \\
\hline $\begin{array}{l}\text { 15. SSy miyy perensos/a para hacer actividata } \\
\text { 15. Hacer actividad fisca cs aburido }\end{array}$ & 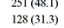 & . $1.09(1.98)$ & $\begin{array}{l}314(438.3) \\
226(59.9)\end{array}$ & $\begin{array}{l}2.81(1.196) \\
1.34(1.58)\end{array}$ & $\begin{array}{r}6.771 \\
223.424\end{array}$ & $\begin{array}{l}0.201 \\
0.100\end{array}$ & $\begin{array}{l}<<.001 \\
<0.001\end{array}$ \\
\hline 16. Me pongo nerivoso'a cuando hago actividad fisica & $122(23.3)$ & $0.37(0.83)$ & $148(60.7)$ & $0.65(1.03)$ & 101.098 & & $<0.001$ \\
\hline
\end{tabular}

Fuente: elaboración propia. 


\section{TABLA 4}

Prevalencia de barreras para la realización de AF y análisis de diferencias en función del cumplimiento de las recomendaciones de práctica de AF y del mismo género

\begin{tabular}{|c|c|c|c|c|c|c|}
\hline \multirow{3}{*}{$\begin{array}{l}\text { Barreras } \\
\text { para la } \\
\text { realización } \\
\text { de } \mathrm{AF}\end{array}$} & & \multirow{3}{*}{$\begin{array}{l}\begin{array}{l}\text { Cumplen las } \\
\text { recomendaciones }\end{array} \\
(\mathrm{n}=524) \\
\mathrm{M}(\mathrm{DT})\end{array}$} & \multirow{3}{*}{$\begin{array}{l}\begin{array}{l}\text { No cumplen las } \\
\text { recomendaciones }\end{array} \\
(\mathbf{n}=377) \\
\text { M(DT) }\end{array}$} & \multicolumn{3}{|c|}{ Contraste entre grupos } \\
\hline & & & & & & \\
\hline & & & & $\mathrm{F}$ & $h^{2} p$ & $\mathbf{p}$ \\
\hline \multirow{3}{*}{$\begin{array}{l}\text { 1. Barreras } \\
\text { contextuales } \\
\text { y de } \\
\text { seguridad } \\
\text { en la } \\
\text { practica }\end{array}$} & Todos & $1.06(1.12)$ & $1.56(1.28)$ & 38.469 & 0.04 & $<0.001$ \\
\hline & Hombres & $0.98(1.01)$ & $1.33(1.28)$ & 6.357 & 0 & 0.012 \\
\hline & Mujeres & $1.17(1.15)$ & $1.63(1.28)$ & 18.032 & 0.02 & $<0.001$ \\
\hline \multirow{3}{*}{$\begin{array}{l}\text { 2. Barreras } \\
\text { referidas al } \\
\text { tiempo }\end{array}$} & & & & & & $<0.001$ \\
\hline & & & & & & \\
\hline & & & & & 0.04 & $<0.001$ \\
\hline \multirow{3}{*}{$\begin{array}{l}\text { 3. Barreras } \\
\text { referidas a } \\
\text { la } \\
\text { capacidad }\end{array}$} & Todos & $0.66(0.77)$ & $1.66(1.16)$ & 237.867 & 0.2 & $<0.001$ \\
\hline & Hombres & $0.48(0.63)$ & $1.37(1.21)$ & 65.905 & 0.06 & 0.003 \\
\hline & Mujeres & $0.92(0.89)$ & $1.75(1.13)$ & 94.561 & 0.09 & $<0.001$ \\
\hline
\end{tabular}

Fuente: elaboración propia.

Por último, este MANOVA (tabla 5) también mostró que en los estudiantes que cumplían las recomendaciones de práctica de AF, existían diferencias significativas en función del género (Lambda de Wilks $=0.977 ; \mathrm{F}(7.079)=\mathrm{p}$ $\left.<0.001 ; \eta^{2} \mathrm{p}=0.023\right)$. En los estudiantes que no cumplían las recomendaciones, también encontramos estas diferencias en función del género (Lambda de Wilks = 0.953; F (14.828) $\left.=\mathrm{p}<0.001 ; \eta^{2} \mathrm{p}=0.047\right)$. La prueba del posthoc de Bonferroni mostró que las mujeres que cumplían las recomendaciones de práctica de AF percibían más barreras que los hombres que cumplían dichas recomendaciones en los factores relativos a la capacidad de disfrute, el tiempo y el contexto y seguridad en la práctica. Asimismo, las mujeres que no cumplían las recomendaciones de práctica de AF percibían más que los hombres que no cumplían dichas recomendaciones en los factores relativos a la capacidad de disfrute y el tiempo (ver tabla 5).
TABLA 5

Prevalencia de barreras para la realización de AF y análisis de diferencias en función del cumplimiento de las recomendaciones de práctica de AF y del género

\begin{tabular}{|c|c|c|c|c|c|c|c|}
\hline \multirow[b]{2}{*}{$\begin{array}{c}\text { Barreras } \\
\text { para la } \\
\text { realización } \\
\text { de AF }\end{array}$} & & \multirow{2}{*}{$\begin{array}{c}\begin{array}{c}\text { Todos } \\
(\mathbf{n}= \\
901)\end{array} \\
\mathrm{M} \\
\text { (DT) }\end{array}$} & \multirow{2}{*}{$\begin{array}{c}\begin{array}{c}\text { Hombres } \\
(\mathbf{n}=\end{array} \\
408) \\
\text { M(DT) }\end{array}$} & \multirow{2}{*}{$\begin{array}{l}\begin{array}{l}\text { Mujeres } \\
(\mathrm{n}=493)\end{array} \\
\text { M (DT) }\end{array}$} & \multicolumn{3}{|c|}{$\begin{array}{c}\text { Contraste entre } \\
\text { hombres y mujeres }\end{array}$} \\
\hline & & & & & $F$ & $h^{2}$ & $p$ \\
\hline \multirow{3}{*}{$\begin{array}{l}\text { 1. Barreras } \\
\text { contextuales } \\
\text { y de } \\
\text { seguridad } \\
\text { en la } \\
\text { practica }\end{array}$} & C & $\begin{array}{l}1.06 \\
(1.12)\end{array}$ & $\begin{array}{l}0.98 \\
(1.01)\end{array}$ & $\begin{array}{l}1.17 \\
(1.15)\end{array}$ & 3.4 & 0 & 0.032 \\
\hline & $\mathrm{NC}$ & $\begin{array}{l}1.56 \\
(1.28)\end{array}$ & $\begin{array}{l}1.33 \\
(1.28)\end{array}$ & $\begin{array}{l}1.63 \\
(1.28)\end{array}$ & 4.637 & 0 & 0.066 \\
\hline & TOT & $\begin{array}{l}1.27 \\
(1.22) \\
\end{array}$ & $\begin{array}{l}1.06 \\
(1.15)\end{array}$ & $\begin{array}{l}1.44 \\
(1.25)\end{array}$ & 21.832 & 0.02 & $<0.001$ \\
\hline \multirow{3}{*}{$\begin{array}{l}\text { 2. Barreras } \\
\text { referidas al } \\
\text { tiempo }\end{array}$} & C & $\begin{array}{l}2.85 \\
(1.54)\end{array}$ & $\begin{array}{l}2.58 \\
(1.51)\end{array}$ & $\begin{array}{l}3.25 \\
(1.49)\end{array}$ & 25.338 & 0.02 & $<0.001$ \\
\hline & $\mathrm{NC}$ & $\begin{array}{l}4.01 \\
(1.54)\end{array}$ & $\begin{array}{l}3.5 \\
(1.83)\end{array}$ & $\begin{array}{l}4.18 \\
(1.19)\end{array}$ & 14.553 & 0.01 & $<0.001$ \\
\hline & TOT & $\begin{array}{l}3.33 \\
(1.64) \\
\end{array}$ & $\begin{array}{l}2.79 \\
(1.63) \\
\end{array}$ & $\begin{array}{l}3.78 \\
(1.5) \\
\end{array}$ & 89.353 & 0.09 & $<0.001$ \\
\hline \multirow{3}{*}{$\begin{array}{l}\text { 3. Barreras } \\
\text { referidas a } \\
\text { la } \\
\text { capacidad } \\
\text { de disfrute }\end{array}$} & C & $\begin{array}{l}0.66 \\
(0.77)\end{array}$ & $\begin{array}{l}0.48 \\
(0.63)\end{array}$ & $\begin{array}{l}0.92 \\
(0.89)\end{array}$ & 23.373 & 0.03 & $<0.001$ \\
\hline & $\mathrm{NC}$ & $\begin{array}{l}1.66 \\
(1.16)\end{array}$ & $\begin{array}{l}1.37 \\
(1.21)\end{array}$ & $\begin{array}{l}1.75 \\
(1.13)\end{array}$ & 11.283 & 0.01 & $<0.001$ \\
\hline & TOT & $\begin{array}{l}1.07 \\
(1.07)\end{array}$ & $\begin{array}{l}0.69 \\
(0.89) \\
\end{array}$ & $\begin{array}{l}1.39 \\
(1.11)\end{array}$ & 107.018 & 0.1 & $<0.001$ \\
\hline
\end{tabular}

\section{Discusión}

El objetivo principal de este estudio fue identificar la prevalencia de barreras de práctica de AF, y analizar las posibles diferencias en función del género y el grado de cumplimiento de las recomendaciones internacionales de AF. En primer lugar, tal y como se había planteado en la primera hipótesis, las barreras externas relativas a la falta de tiempo parecen el principal obstáculo para la realización de AF entre los estudiantes universitarios. Estos resultados están en sintonía con los encontrados en trabajos precedentes en Reino Unido (Lovell et al., 2010), Arabia Saudí (Gawwad, 2008), Egipto (El-Gilany et al., 2011), Brasil (Ferreira et al., 2012), Turquía (Arzu, Handan, \& Eker, 2006), y también en España (Capdevila et al., 2007; Gómez-López et al., 2010; Martínez-Lemos et al., 2014.). De manera específica, las causas de esa falta de 
tiempo se deben a un exceso de trabajos en la universidad, a la alta cantidad de materias a estudiar, y a las tareas domésticas que se deben realizar en casa. Una posible explicación a los resultados encontrados puede encontrarse en el hecho de que la entrada en la etapa universitaria está asociada en muchos casos, a cambios sustanciales en el estilo de vida de muchos de los estudiantes, que deben asumir más responsabilidades (e.g. cambio de la residencia habitual, asunción de tareas del hogar, además de las tareas académicas, compromisos familiares, etc.) (Daskapan et al., 2006; Ibrahim et al., 2013). En esta misma línea, un estudio realizado en el contexto universitario en España, señaló que la exigencia de los estudios, la falta de tiempo libre y la incompatibilidad de los horarios con las ocupaciones diarias, pueden ser algunos de los factores que causan una menor práctica de AF (Pavón \& Moreno, 2008). De manera complementaria, el análisis de regresión logística binaria indicó que las barreras relativas al tiempo predecían significativamente el cumplimiento de las recomendaciones de AF. A tenor de los resultados encontrados, la alta prevalencia de barreras relativas a la falta de tiempo en la etapa universitaria, es especialmente preocupante ya que algunos estudios han señalado que puede dar lugar al abandono de la práctica de AF (GomézLópez et al., 2011).

Seguidamente, otras barreras externas como las relativas al contexto y seguridad en la práctica también mostraron una alta prevalencia, concretamente las barreras relativas a "No tengo a nadie con quien realizar actividad física" y "No tengo el equipo necesario para hacer actividad física". En este sentido, la falta de adecuación entre la oferta y la demanda deportiva dentro de los programas universitarios pueden suponer que las actividades no estén adaptadas a las preferencias e intereses de algunos estudiantes, que llegan a percibir que no tienen compañeros con los que realizar AF o el material disponible para desempeñar la actividad (Pavón \& Moreno, 2008; Rodríguez et al., 2013). La prevalencia de este tipo de barreras está en línea con los resultados hallados en otros estudios previos en Malasia (Ibrahim et al., 2013) y Brasil (Reichert et al., 2007), aunque el mal tiempo o la falta de dinero o de instalaciones para practicar AF se mostraron como las principales barreras percibidas en estos países. Por tanto, en consonancia con lo expuesto en la literatura científica, las barreras externas parecen mostrar una mayor prevalencia que las barreras internas para la práctica de AF en los estudiantes universitarios (Daskapan et al., 2006).

Finalmente, aunque las barreras internas relativas a la capacidad de disfrute obtienen puntuaciones más que bajas que el resto, algunos ítems como "Por lo general estoy cansado/a para hacer actividad física", "No soy bueno en las actividades físicas y/o deportivas" y "Soy muy perezoso/a para hacer actividad física" mostraron valores más altos que todos los ítems relativos al contexto y seguridad en la práctica. Estos resultados se encuentran en sintonía com otros estudios realizados en población universitaria, en los que la falta de habilidades fue identificada como la principal barrera para realizar AF (Ramírez-Vélez et al., 2015). Por otro lado, el cansancio o la pereza fueron dos de las barreras internas más puntuadas, en línea con los hallazgos encontrados en otros estudios en población universitaria en España (GómezLópez et al., 2010; Martínez-Lemos et al., 2014), Chile (Rodríguez et al., 2013) o Malasia (Ibrahim et al., 2013). Algunos autores exponen que la pereza y el cansancio son razones intrínsecas de tipo personal que pueden estar relacionadas, entre otros factores, con una baja condición física entre los universitarios, o al malestar derivado de una posible mala experiencia de la actividad en sus inicios (Niñerola, Capdevila, \& Pintanel, 2006). En esta línea, un estudio longitudinal en población universitaria reveló que la condición física de los estudiantes empeoraba progresivamente (Pribis, Burtnack, McKenzie, \& Thayer, 2010). Por tanto, parece importante promover actitudes y hábitos positivos desde la niñez y la adolescencia, en relación a la práctica de $\mathrm{AF}$, con objeto de inhibir esta barrera para su realización en la etapa jovenadulta.

De manera complementaria, y en sintonía con otros estudios (Gunnell et al., 2015), este 
tipo de barreras relativas a la capacidad de disfrute predijeron negativamente los niveles de AFMV. Por tanto, parece muy importante disminuirlas para conseguir un estilo de vida más activo en la población universitaria. Siguiendo la teoría de la autodeterminación (Ryan \& Deci, 2000), este hecho puede deberse a que si los estudiantes no se sienten competentes, tienen una menor probabilidad de divertirse o de mostrar una mayor persistencia en la práctica de AF (Cocca, Mayorga-Vega, \& Viciana, 2013; Inchley, Kirby \& Currie, 2011; Kulavic, Hultquist, \& McLester, 2013). Estos resultados sugieren que la percepción de competencia de competencia puede ser una necesidad psicológica básica influyente en la percepción de barreras para la realización de AF (Samperio, JiménezCastuera, Lobato, Leyton, \& Claver, 2016). Así, el apoyo de este antecedente social se muestra como una pieza clave en el diseño e implementación de programas de intervención que puedan favorecer el disfrute y mejorar los niveles de AFMV (Bean, Harlow, \& Kendellen 2017).

La segunda hipótesis sostuvo que los estudiantes universitarios que no cumplían las recomendaciones de práctica de $\mathrm{AF}$, percibirían más barreras para realizar $\mathrm{AF}$ que aquellos que sí cumplían con dichas recomendaciones. En este sentido, los estudiantes universitarios que no cumplieron las recomendaciones de práctica de AF presentaron valores significativamente superiores en la mayoría de barreras analizadas, a excepción de los ítems "No hay un buen sitio para hacer actividad física" y "No es seguro estar haciendo ejercicio físico en la calle, barrio, parque...", por lo que la hipótesis se cumplió en su mayor parte. Estos resultados están en línea del estudio de Awadalla et al. (2014), en el que los estudiantes universitarios que no cumplían las recomendaciones de AF reportaron valores significativamente superiores en la mayoría de barreras analizadas. No obstante, otros autores señalaron que estas diferencias se aprecian en función de la tipología de barreras que se analiza. Así, en sintonía con este estudio, Martínez-Lemos et al. (2014), en una muestra de universitarios españoles, señaló que en las barreras relativas a la fatiga/pereza y a las obligaciones, existían diferencias significativas entre los estudiantes que cumplían y no cumplían las recomendaciones de práctica de $\mathrm{AF}$. De igual modo, en este último estudio, en las barreras relativas al contexto no hubo diferencias significativas entre unos estudiantes y otros, tal y como ocurre en el presente trabajo. Este hecho puede deberse a que los sujetos que perciben más barreras relativas a la capacidad de disfrute y a la falta de tiempo, tienen menos posibilidades de mantener un estilo de vida activo (RamírezVélez et al., 2015). Por tanto, parece un aspecto clave adecuar los programas de intervención a las características de la muestra y el contexto estudiado, tratando de reducir el número de barreras para desempeñar la práctica de AF.

Finalmente, la tercera hipótesis señaló que las mujeres universitarias percibirían más barreras de práctica de AF que los hombres universitarios, lo que se confirma a raíz de los resultados encontrados. De este modo, las mujeres que cumplían las recomendaciones de AF presentaron valores superiores en todas las barreras analizadas que los hombres que cumplían dichas recomendaciones. En esta misma línea, las mujeres que no cumplían las recomendaciones de AF presentaron valores superiores en las barreras referentes al tiempo y a la capacidad de disfrute que los hombres que no cumplían dichas recomendaciones. Estos resultados son congruentes con otros estudios en universitarios en los que las mujeres presentaban una mayor prevalencia de barreras que los hombres en falta de tiempo, falta de habilidades y en el contexto de práctica (Martínez-Lemos et al., 2014; Ramírez-Vélez et al., 2015). Estos resultados pueden ser debidos a que las mujeres suelen sentirse menos competentes en las clases de EF a lo largo de la etapa infantil y adolescente, por lo que no obtienen experiencias muy positivas, lo que pueden generar más barreras referentes a la capacidad de disfrute en la etapa joven adulta (Jose, Blizzard, Dwyer, McKercher, \& Venn, 2011; Mitchell, Gray, \& Inchley, 2013). Otra posible justificación a los hallazgos encontrados en las barreras referentes al tiempo, es que las mujeres universitarias le 
otorgan una mayor importancia a su tiempo de ocio y otras actividades sociales y aficiones personales, a diferencia de los hombres, que suelen decantarse por actividades más deportivas (Pavón \& Moreno, 2008). Por último, en las barreras referentes al contexto y la seguridad en la práctica, las mujeres pueden tener una mayor prevalencia debido a la falta de apoyo de sus iguales, que realizan una menor práctica de AF que los hombres, o al miedo o inseguridad que manifiestan algunas mujeres en sus calles o barrios (Ferret, Baylina, \& Ortiz, 2012). Por tanto, en líneas generales, el género parece una variable relevante en la percepción de barreras para la práctica de AF. Así, la mayor prevalencia de barreras por parte de las mujeres, quizá sea uno de los factores que pueden explicar que el género femenino realice una menor práctica de AF y alcance un menor cumplimiento de las recomendaciones de práctica de la misma (Rodríguez et al., 2013; Varela-Mato et al., 2012). Por otro lado, no se observaron diferencias en el grado de importancia de los diferentes tipos de barreras, en donde las relativas al tiempo son las que aparecen con una mayor frecuencia en ambos géneros, tal y como señalan otros estudios en España (Gómez-López et al., 2011).

Por tanto, los resultados encontrados arrojan numerosas implicaciones prácticas para el diseño de programas de intervención en estudiantes universitarios. A continuación, en base a los resultados encontrados, se enumeran algunas acciones específicas que se podrían desarrollar: a) Promover el transporte activo a la universidad caminando o en bicicleta, b) Ofertar actividades deportivas y recreativas que respondan a los intereses y preferencias de los universitarios, y tengan varios niveles de progresión, c) Difundir los eventos deportivos que van a realizarse en el contexto próximo a través de las redes sociales (e.g. carrera popular contra el cáncer de mama), d) Facilitar un horario flexible de las instalaciones deportivas así como del material deportivo para que puedan ser utilizadas en todo momento, e) Realizar descuentos en la utilización de instalaciones públicas (e.g. gimnasios, piscinas, etc.). Para ello, se debe prestar atención a los intereses y características de las mujeres y personas más inactivas, dado que presentan una alta prevalencia de barreras de práctica de AF. El empoderamiento de todos los agentes y sectores que influyen en la práctica de AF de los estudiantes universitarios parece necesario para mejorar los niveles de AFMV (Plotnikoff et al., 2015).

Las principales limitaciones del estudio pueden residir en los instrumentos de medida utilizados para cuantificar los niveles de AFMV y la percepción de barreras, a través de instrumentos autoadministrados. En este sentido, algunos estudios señalan que a pesar de la validez del IPAQ-SF, este instrumento puede sobreestimar los resultados hallados. No obstante, su uso está justificado en este estudio debido al número de estudiantes de la muestra. En futuros estudios parece oportuno dar soporte empírico a los hallazgos encontrados mediante la utilización de acelerómetros u otros instrumentos de medida más objetivos. Asimismo, la utilización de metodología cualitativa puede ayudar a justificar algunos de los resultados hallados, aportando una mayor información sobre las barreras percibidas para la realización de $\mathrm{AF}$ en el contexto universitario. De igual modo, el estudio de las barreras de práctica de AF en función del nivel socioeconómico, el tipo de carrera universitaria o el curso académico podría aportar más información en este grupo poblacional. Otra prospectiva de estudio podría ser complementar los resultados hallados mediante la inclusión de otras variables presentes dentro del modelo Social-Ecológico y otras teorías motivacionales (e.g. teoría de la autodeterminación), que podrían aportar una visión más amplia de los factores que dificultan u obstaculizan la realización de AF en los estudiantes universitarios (e.g. motivación autodeterminada, experiencias previas en las clases de EF, intención de práctica futura). Por otro lado, el diseño, aplicación y evaluación de programas de intervención multicomponente y políticas para la promoción de la $\mathrm{AF}$ en los estudiantes universitarios se abren como líneas prioritarias de actuación. 


\section{Conclusiones}

Los estudiantes universitarios identificaron un gran número barreras para la práctica de $\mathrm{AF}$. De acuerdo a los resultados obtenidos, la percepción de falta de tiempo se mostró como uno de los mayores obstáculos para realizar AF en la población universitaria. Por otro lado, las mujeres y las personas que no cumplían las recomendaciones de $\mathrm{AF}$ presentaron una alta prevalencia de barreras de práctica de AF. En base a los resultados encontrados se destaca la importancia de desarrollar programas y estrategias de intervención, que permitan mejorar la gestión del tiempo de ocio y la motivación de los estudiantes universitarios para reducir las barreras de práctica de AF y asentar estilos de vida más activos y autónomos en esta etapa.

\section{Agradecimientos}

Este estudio se ha realizado gracias a la aportación de la Consejería de Empleo, Empresa e Innovación del Gobierno de Extremadura a través de los fondos europeos de Desarrollo Regional. Nos gustaría agradecer la participación a todos los estudiantes del Campus de Cáceres, perteneciente a la Universidad de Extremadura, y a sus respectivos profesores por facilitarnos la recogida de los datos.

\section{Referencias}

Arias-Palencia, N.M., Solera-Martínez, M., Gracia-Marco, L., Silva, P., MartínezVizcaíno, V., Cañete-García-Prieto, J., \& Sánchez-López, M. (2015). Levels and Patterns of Objectively Assessed Physical Activity and Compliance with Different Public Health Guidelines in University Students. PLoS One, 10(11). https://doi.org /10.1371/journal.pone.0141977.

Arzu, D., Handan, E., \& Eker, L. (2006). Perceived barriers to physical activity in university students. Journal of Sports Science and Medicine, 5(4), 615-20.

Ashton, L. M., Hutchesson, M. J., Rollo, M. E., Morgan, P. J., \& Collins, C. E. (2016). Motivators and Barriers to Engaging in Healthy Eating and Physical Activity A Cross-Sectional Survey in Young Adult Men. American Journal of Men's Health, 11(2), 1-14.

Awadalla, N.J., Aboelyazed, A.E., Hassanein, M.A., Khalil, S.N., Aftab, R., Gaballa, I.I., \& Mahfouz A.A. (2014). Assessment of physical inactivity and perceived barriers to physical activity among health college students, south-western Saudi Arabia. East MediterrHealth J, 20 (10), 596-604.

Bean, C., Harlow, M., \& Kendellen, K. (2017). Strategies for fostering basic psychological needs support in high quality youth leadership programs. Evaluation and Program Planning, 61, 76-85.

Booth, M. L. (2000). Assessment of Physical Activity: An International Perspective. Research Quarterly for Exercise and Sport, 71(2), 114-120.

Bray, S. R. (2007). Self-efficacy for coping with barriers helps students stay physically active during transition to their first year at a university. Research Quarterly for Exercise \&? Sport, 78(2), 61-70.

Capdevila, L., Niñerola, J., Cruz, J., Losilla, J. M., Parrado, E., Pintanel, M. ... \& Vives, J. (2007). Exercise motivation in university community members: A behavioural intervention. Psicothema, 19, 250-255.

Chinn, D. J., White, M., Harland, J., Drinkwater, C., \& Raybould, S. (1999). Barriers to physical activity and socio-economic position: implications for health promotion. Journal of Epidemiology and Community Health, 53(3), 191-192.

Clemente, F.M., Nikolaidis, P.T., Martins, F.M., \& Mendes, R.S. (2016). Physical Activity Patterns in University Students: Do They Follow the Public Health Guidelines? PLoS One, 11(3). https://doi.org/10.1371/journal .pone. 0152516 . 
Cocca, A., Liukkonen, J., Mayorga-Vega, D., \& Viciana-Ramírez, J. (2014). Health-related physical activity levels in spanish youth and young adults. Perceptual $\mathbb{E}$ Motor Skills: Physical Development $\mathbb{E}$ Measurement, 118(1), 247-260.

Cocca, A., Mayorga-Vega, D., \& Viciana, J. (2013). Relación entre niveles de actividad física y placer de ser activos en estudiantes universitarios. Revista Iberoamericana de Psicología del Ejercicio y el Deporte, 8(2), 359-372.

Craig, C. L., Marshall, A. L., Sjostrom, M., Bauman, A. E., Booth, M. L., Ainsworth, B. E., ... \& Oja, P. (2003). International physical activity questionnaire: 12 -country reliability and validity. Medicine and Science in Sports and Exercise, 35 (8), 1381-1395.

Daskapan, A., Tuzun, E. H., \& Eker, L. (2006). Perceived barriers to physical activity in university students. Journal of Sports Science and Medicine, 5(4), 615-620.

Deliens, T., Deforche, B., De Bourdeaudhuij, I., \& Clarys, P. (2015). Determinants of physical activity and sedentary behaviour in university students: a qualitative study using focus group discussions. BMC Public Health, 15(1), 201.

El-Gilany, A. H., Badawi, K., El-Khawaga, G., \& Awadalla, N. (2011). Physical activity profile of students in Mansoura University, Egypt. Eastern Mediterranean Health Journal, 17(8), 694-702.

Ferreira, T., Aparecido, S., \& Rodrigues, A. (2012). Perceived barriers by university students in relation the leisure-time physical activity. Brazilian Journal of Kinanthropometry and Human Performance, 15(2), 164-173.

Ferret, M. P., Baylina, M., \& Ortiz, A. (2012). Los lugares de la amistad y la vida cotidiana de chicas y chicos adolescentes en un barrio de Barcelona. Revista Latino-Americana de Geografia e Gênero, 3(2), 116-124.

Gawwad, E.S. (2008). Stages of Change in Physical Activity, Self-Efficacy and Decisional Balance among Saudi University
Students. Journal of Family and Community, 15, 107-115.

Gómez-López, M., Granero-Gallegos, A., \& Baena-Extremera, A. (2010). Perceived barriers by university students in the practice of physical activities. Journal of Sports Science and Medicine, 9(3), 374-381.

Gómez-López, M., Granero-Gallegos, A., BaenaExtremera, A., \& Ruiz-Juan, F. (2011). The abandonment of an active lifestyle within university students: reasons for abandonment and expectations of reengagement. Psychologica Belgica, 51(2), 155-175.

Gunnell, K. E., Brunet, J., Wing, E. K., \& Bélanger, M. (2015). Measuring Perceived Barriers to Physical Activity in Adolescents. Pediatric Exercise Science, 27(2), 252-261.

Gyurcsik, N. C., Spink, K. S., Bray, S. R., Chad, K., \& Kwan, M. (2006). An ecologically based examination of barriers to physical activity in students from grade seven through first-year university. The Journal of Adolescent Health, 38(6), 704-711.

Haase, A., Steptoe, A., Sallis, J. F., \& Wardle, J. (2004). Leisure-time physical activity in university students from 23 countries: associations with health beliefs, risk awareness, and national economic development. Preventive Medicine, 39(1), 182-190.

Han, J. L., Dinger, M. K., Hull, H. R., Randall, N. B., Heesch, K. C., \& Fields, D. A. (2008). Changes in women's physical activity during the transition to college. American Journal of Health Education, 39(4), 194-199.

Hazam, A. (2013). Measuring Stages of Change, Perceived Barriers and Self efficacy for Physical Activity in Saudi Arabia. Asian Pacific Organization for Cancer Prevention, 14(2), 1009-1016.

Ibrahim, S., Karim, N. A., Oon, N. L., \& Ngah, W. Z. (2013). Perceived Physical Activity Barriers Related to Body Weight Status and Sociodemographic Factors among Malaysian Men in Klang Valley. BMC Public Health, 13, 236-246. 
Inchley, J., Kirby, J., \& Currie, C. (2011). Longitudinal changes in physical selfperceptions and associations with physical activity during adolescence. Pediatric Exercise Science, 23(2), 237-249.

Jose, K. A., Blizzard, L., Dwyer, T., McKercher, C., \& Venn, A. J. (2011). Childhood and adolescent predictors of leisure time physical activity during the transition from adolescence to adulthood: a population based cohort study. International Journal of Behavioral Nutrition and Physical Activity, $8(54)$.

Keating, X. D., Guan, J., Piñero, J. C., \& Bridges, D. M. (2005). A meta-analysis of college student physical activity levels. Journal of American College Health, 54(2), 116-215.

Kulavic, K., Hultquist, C. N., \& McLester, J. R. (2013). A comparison of motivational factors and barriers to physical activity among traditional versus nontraditional college students. Journal of American College Health, 61(2), 60-66.

Lovell, G., El Ansari, W., \& Parker, J. K. (2010). Perceived Exercise Benefits and Barriers of Non-Exercising Female University Students in the UK. International Journal of Environmental Research and Public Health, 7(3), 784-798.

Martínez-Lemos, R., Puig-Ribera, A., \& GarcíaGarcía, O. (2014). Perceived barriers to Physical Activity and related factors in Spanish University students. Open Journal of Preventive Medicine, 4(4), 164-174.

Mitchell, F., Gray, S., \& Inchley, J. (2013). 'This choice thing really works ...' Changes in experiences and engagement of adolescent girls in physical education classes, during a school-based physical activity programme. Physical Education and Sport Pedagogy, 20(6), 593-611.

Niñerola, J., Capedvila, L., \& Pintanel, M. (2006). Barreras percibidas y actividad física: el autoinforme de barreras para la práctica de ejercicio físico. Revista de Psicología del Deporte, 15(1), 53-69.

Organización Mundial de la Salud [OMS] (2010). Recomendaciones mundiales sobre actividad física para la salud. Ginebra, Suiza. Recuperado de: http://apps.who.int/iris/bitstream/1066 5/44441/1/9789243599977_spa.pdf

Pavón, A., \& Moreno, J. A. (2008). Actitud de los universitarios ante la práctica físicodeportiva: Diferencias por géneros. Revista de Psicología del Deporte. 17(1), 7-23.

Plotnikoff, R. E., Costigan, S. A., Williams, R. L., Hutchesson, M. J., Kennedy, S. G., ...., \& Germov, J. (2015). Effectiveness of interventions targeting physical activity, nutrition and healthy weight for university and college students: a systematic review and meta-analysis. International Journal of Behavioral Nutrition and Physical Activity, 12,45 .

Pribis, P., Burtnack, C., McKenzie, S., \& Thayer, J. (2010). Trends in body fat, body mass index and physical fitness among male and female college students. Nutrients, 2(10), 1075-1085.

Ramírez-Vélez, R., Tordecilla-Sanders, A., Laverde, D., Hernández-Novoa, J. G., Ríos, M., .. \& M Martínez-Torres, J. (2015). The prevalence of barriers for Colombian college students engaging in physical activity. Nutrición Hospitalaria. $31(2), 858-865$.

Reichert, F. F., Barros, A. J. D., Domingues, M. R., \& Hallal, P. C. (2007). The role of perceived personal barriers to engagement in leisuretime physical activity. American Journal of Public Health, 97(3), 515-519.

Rodríguez, F., Palma, X., Romo, A., Escobar, D., Aragú, B., Espinoza, L., .. \& Gálvez, J. (2013). Hábitos alimentarios, actividad física y nivel socioeconómico en estudiantes universitarios de Chile. Nutrición Hospistalaria, 28(2), 447-455.

Rodríguez-Muñoz, S., Corella, C., Abarca-Sos, A., \& Zaragoza, J. (2017). Validation of three short physical activity questionnaires with accelerometers among university students in Spain. The Journal of Sports Medicine and Physical Fitness. Recuperado de: https://www.ncbi.nlm.nih.gov/pubmed/ 28249383 
Román, B., Ribas, L., Ngo, J., \& Serra, L. (2013). Validación en población catalana del cuestionario internacional de actividad física. Gaceta Sanitaria, 27(3), 254-257.

Ryan, R. M., \& Deci, E. L. (2000). Selfdetermination theory and the facilitation of intrinsic motivation, social development, and well-being. The American Psychologist, 55(1), 68-78.

Sallis, J. F., Owen, N., \& Fisher, E. B. (2008). Ecological models of health behavior, in: Glanz, K.,Rimer, B. K., \& Viswanath, K. (Eds.). Health behavior and health education: theory, research, and practice (pp. : 465-486). 4th. CA: Jossey-Bass, San Francisco.

Samperio, J., Jiménez-Castuera, R., Lobato, S., Leyton, M., \& Claver, F. (2016). Variables motivacionales predictoras de las barreras para la práctica de ejercicio físico en adolescentes. Cuadernos de Psicología del Deporte, 16(2), 65-76.

Serrano-Sánchez, J. A., Lera-Navarro, A., Dorado-García, C., GonzálezHenriquez, J. J., \& Sanchis-Moysi, J. (2012). Contribution of Individual and Environmental Factors to Physical Activity Level among Spanish Adults. PLoS ONE, 7(6), e38693.

Spence, J. C., \& Lee, R. E. (2003). Toward a comprehensive model of physical activity. Psychology of Sport and Exercise, 4(1), 7-24.

Varela-Mato, V., Cancela, J. M., Ayan, C., Martín, V., \& Molina, A. (2013). Lifestyle and Health among Spanish University Students: Differences by Gender and Academic Discipline. International Journal of Environmental Research and Public Health, 10(8), 2728-2741.

Yan, A., Voorhees, C. C., Beck, K. H., \& Wang, M. (2014). A social ecological assessment of physical activity among urban adolescents. American Journal of Health Behavior, 38(3), 379-391.

Zaragoza, J., Generelo, E., Julián, J. A., \& AbarcaSos, A. (2011). Barriers to participation in physical activity among adolescent girls defined by physical activity level. The
Journal of Sports Medicine and Physical Fitness, 51(1), 128-135.

\section{Notas}

* Artículo de investigación. 